\title{
Propiedades balísticas de perdigones antidisturbios "menos letales" y su relación con trauma ocular severo en Chile
}

\author{
Ballistic properties of "less-lethal" shotgun pellets and severe ocular trauma in Chile
}

\author{
Scott A. Reynhout ${ }^{1}$
}

Resumen: Se analiza el comportamiento balístico de dos tipos de perdigones "menos letales" usados por las fuerzas policiales en Chile para entender mejor los factores fundamentales que conllevan a la inusual cifra de lesiones oculares en el marco de las manifestaciones masivas ocurridas en Chile desde octubre de 2019. Para evaluar los riesgos de penetración y trauma ocular severo, se construyeron curvas de energía normalizada (E/a) en base a información publicada por las fuerzas policiales y fabricantes de municiones. Aun cuando el riesgo asociado al uso de las municiones según protocolos es leve, este artículo presenta que de todas formas existe riesgo de trauma ocular severo en todas las distancias de uso. La balística de los perdigones, combinada con la imprecisión de municiones multiproyectiles, son factores importantes para explicar la alta incidencia de trauma ocular severo. El caso de Chile es parte de un aumento global en la incidencia de lesiones oculares causadas por municiones consideradas menos letales, lo cual demanda una reevaluación de las políticas que regulan su uso.

Palabras clave: balística; menos-letal; perdigones; Carabineros; Chile; proyectil de impacto cinético.

Abstract: The ballistic characteristics of two types of "less-lethal" multi-projectile shotgun rounds used by law enforcement in Chile are analyzed to better understand their contribution to the unusually high occurrence of ocular injuries at mass demonstrations since October 2019. Normalized energy (E/a) curves are constructed using publicly-available information from law enforcement and the manufacturers of the munitions to evaluate the risks of skin penetration and severe ocular trauma. Although the risk of penetration is small when these munitions are used according to local protocols, the risk of severe ocular trauma exists at all distances of approved use, and the ballistics of these pellets-along with the imprecision of multi-projectile shotgun rounds-help to explain the high incidence of severe ocular trauma. The example of Chile is part of a worldwide acceleration in the incidence of ocular injuries by "less-lethal" munitions and demands a reevaluation of their suitability for crowd control.

Keywords: ballistics; less-lethal; pellets; Carabineros; Chile; kinetic impact projectile.

Fecha de envío: 15 de febrero de 2020 - Fecha de aceptación: 17 de junio de 2020

\section{Introducción}

Una de las principales características de las manifestaciones masivas que se han desarrollado en Chile desde el 18 de octubre de 2019 ha sido la alta incidencia de casos de trauma ocular severo (TOS) por impacto de perdigones "de goma". A lo largo del país, en el periodo comprendido entre el 19 de octubre y el 8 de noviembre de 2019, los impactos de balines antidisturbios se han visto involucrados en 135 casos de herida penetrante ocular (HPO), estallido ocular, cuerpos extraños intraorbitarios y trauma ocular contuso
(Rodríguez et al., 2019). Puesto en contexto, durante el periodo de 1990-2017 el uso de municiones "no letales" o "menos letales" estuvo involucrado en al menos 310 casos de lesiones oculares en todo el mundo (Haar et al., 2017). Chile está pronto a superar esta cifra en tan solo meses.

Las armas menos letales se utilizan mundialmente como instrumentos de control de manifestaciones por las fuerzas policiales. Aunque no existe una definición común aceptada referente a este tipo de armas (United Nations Regional Centre for Peace, Disarmament and

(1) Departamento de Geología, Facultad de Ciencias Físicas y Matemáticas, Universidad de Chile, Santiago, Chile.

Autor de correspondencia: scott.a.reynhout@gmail.com 
Development in Latin America and the Caribbean, 2016), Carabineros de Chile dice utilizar varios "dispositivos, armas, y/o municiones no letales o menos letales" para responder a disturbios públicos. Los protocolos que rigen el uso de la fuerza de Carabineros autoriza la utilización de proyectiles de impacto cinético menos letales (kinetic impact projectiles, o KIP), en particular, cartuchos multiproyectiles que contienen 12 perdigones "de goma" de $8 \mathrm{~mm}$ de diámetro, hechos por la empresa chilena TEC Harseim (Figura 1a; Ministerio del Interior y Seguridad Pública, 2019). La Policía de Investigaciones (PDI) utiliza otro tipo de cartucho multiproyectil, producidos por la Companhia Brasileira de Cartuchos (CBC), que contienen 3 perdigones de mayor tamaño (Companhia Brasileira de Cartuchos [CBC], 2012; Bellolio et al., 2019). El uso de estos perdigones ha sido reportado recientemente en el marco de las protestas en Santiago (Figura 1b; Figueroa \& Romero, 2020).
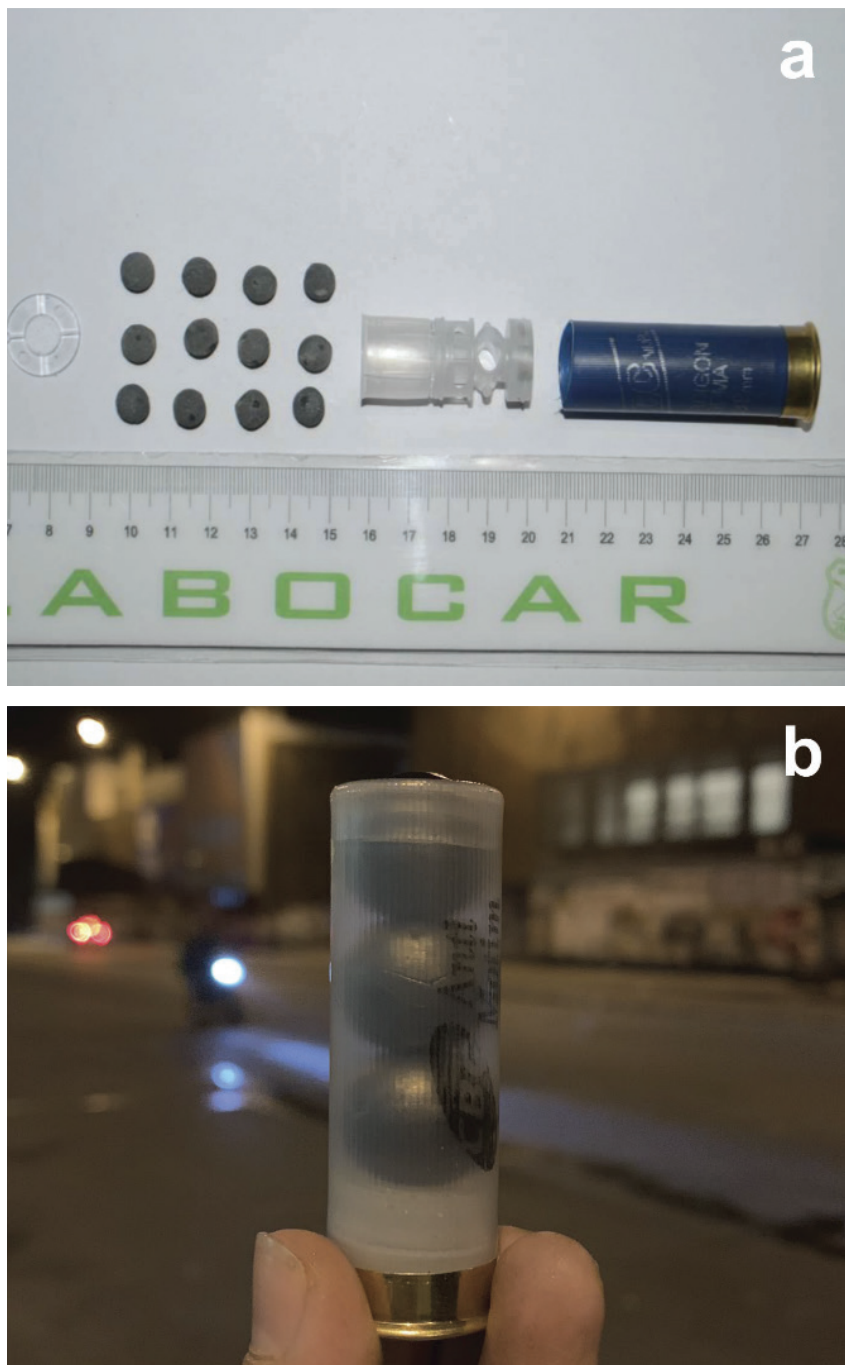

Figura 1: (a) Cartucho y perdigones de las municiones antidisturbios marca TEC Harseim (Labocar, 2019). (b) Cartucho y perdigones de las municiones antidisturbios marca CBC (Figueroa \& Romero, 2020):
Desde la década de 1970, diversos estudios han identificado riesgos de los KIP_tales como contusiones, laceraciones, fracturas óseas, lesiones oculares y muerte-en balas de goma (Millar et al., 1975), balas plásticas (Rocke, 1983), balas metálicas con una capa de goma (Mahajna et al., 2002) y balas bean bag (de Brito et al., 2001), entre otros. Sin embargo, hasta donde el autor tiene conocimiento, no existen antecedentes en la literatura médica ni científica sobre los efectos de perdigones similares a los utilizados en las manifestaciones en Chile.

La falta de información sobre las municiones ha dado lugar a varios informes sobre la composición de los perdigones antidisturbios (Corgne, 2019; Cox et al., 2019; Jorquera \& Palma, 2019; Lazo, 2019), e incluso una respuesta por parte de Carabineros (Labocar, 2019). Sin embargo, la mayoría de los informes ya publicados se enfocan principalmente en la composición de los proyectiles. Ninguno de ellos se refiere al comportamiento de estos proyectiles en vuelola balística de los proyectiles-que es el punto más relevante en términos de lesiones oculares.

Dado que estas municiones siguen siendo usadas habitualmente por las fuerzas policiales en Chile, es de interés público saber más sobre las armas estatales, específicamente, los perdigones antidisturbios. Este informe tiene el objetivo de cuantificar el comportamiento balístico de los proyectiles en uso, determinar los riesgos de estos proyectiles, y posicionarlos dentro de la literatura ya existente de municiones menos letales. Se usa información publicada por los usuarios y/o fabricantes para reconstruir la energía efectiva de los proyectiles en función de la distancia, y a través de este análisis, evaluar los riesgos asociados con las distancias sugeridas en los protocolos de uso de las municiones antidisturbios.

\section{¿Cómo funcionan los KIP?}

Como cualquier proyectil, los KIP funcionan en base a la transferencia de energía cinética desde un proyectil a un blanco. El KIP ideal tiene un perfil balístico que permitiría una entrega suficiente de energía cinética para disuadir o incapacitar al blanco, sin generar riesgo de discapacidad permanente ni lesiones fatales. La energía cinética de un proyectil se obtiene mediante la siguiente formula:

$$
C E=\frac{1}{2} m v^{2}
$$

donde $m=$ la masa del proyectil, y $v=$ la velocidad instantánea del proyectil. 
En el contexto balístico, también es importante considerar el área efectiva del proyectil para determinar los efectos dañinos de éstos. Un proyectil cuya energía cinética está dispersada sobre un área pequeña tendrá una capacidad penetrativa mayor - y por ende mayor letalidad-que un proyectil con la misma energía cinética dispersada sobre un área grande.

A diferencia de las municiones letales, el diseño de los KIP intenta reducir la probabilidad de penetración en el cuerpo, mediante cambios en la masa, velocidad o área efectiva del proyectil. Los perdigones antidisturbios TEC tienen las mismas dimensiones que las municiones metálicas de caza $(\mathrm{d}=0,8 \mathrm{~cm}$, equivalente a \#0 buckshot), pero su composición menos densa y menor velocidad disminuye la energía cinética terminal de los perdigones. Los perdigones antidisturbios $\mathrm{CBC}$ tienen cinco veces más masa que los perdigones de TEC, pero su tamaño grande $(\mathrm{d}=1,8 \mathrm{~cm})$ aumenta el área efectiva del proyectil, lo cual implica un menor potencial penetrativo.

Un informe interno de Carabineros advirtió riesgos cualitativos de las municiones de TEC Harseim que incluyó estallido ocular y potencial muerte, aunque no es claro en qué medida se difundieron estos conocimientos dentro de la institución (Carabineros de Chile, 2012). Oficiales de la PDI testificaron frente a la Cámara de Diputados que un peritaje interno determinó que los perdigones
CBC "tienen [sic] una letalidad mucho menor" (Bellolio et al., 2019), pero el contenido de dicho informe ya no es público.

\section{Metodología}

Se evaluaron los riesgos asociados a los dos tipos de perdigones antidisturbios en uso activo por Carabineros de Chile en función de la distancia recorrida desde salida del cañón. Se confeccionaron curvas que muestran la evolución de la energía cinética de los perdigones de acuerdo a la distancia, y de este modo se identificaron distancias donde existe una alta probabilidad de ocurrencia de lesiones graves.

Para comprobar los efectos de impactos de proyectiles en la superficie corporal varios estudios han utilizado el cociente entre energía y área (E/a), también conocido en la literatura como normalized energy o energy density (DiMaio, 1981):

$$
\frac{E}{a}=\frac{m v^{2}}{2 \pi r^{2}}
$$

donde $m$ = la masa del proyectil, $v$ = la velocidad instantánea del proyectil, y $r=$ el radio seccional del proyectil. Estudios previos han determinado experimentalmente riesgos de penetración de la piel y lesiones oculares para definir valores umbrales y probabilísticos de E/a (Tablas 1 y 2 ).

Tabla 1: Valores empíricos de E/a asociados con riesgos de penetración de la piel:

\begin{tabular}{llccl} 
Riesgo & Proyectil & Diámetro (cm) & E/a (J cm ${ }^{-2}$ ) Fuente \\
\hline Penetracion de la piel, umbral & Esfero de plomo & 1,125 & 21,3 & Journee (1907) \\
Penetracion de la piel, umbral & Esfero de plomo & 0,85 & 20,6 & Matoo (1974) \\
Penetracion de la piel, umbral & Bala de plomo, calibre .38 & 0,91 & 19,5 & DiMaio (1981) \\
Penetracion de la piel, umbral & Balín de plomo, diabolo & 0,45 & 18,4 & DiMaio (1981) \\
Penetracion de la piel, umbral & Balín de plomo, diabolo & 0,56 & 13,0 & DiMaio (1981) \\
Penetracion de la piel, umbral & Esfero de acero & 0,45 & 18 & Rathman (1984) \\
Penetracion de la piel, umbral & Bala de plomo, puntiagudo & 0,45 & 25 & Rathman (1984) \\
Penetracion de la piel, umbral & Bala de plomo, punto plano & 0,45 & 30 & Rathman (1984) \\
Penetracion de la piel, umbral & Bala de plomo, puntiagudo & 0,56 & 26 & Rathman (1984) \\
Penetracion de la piel, umbral & Bala de plomo, punto plano & 0,56 & 39 & Rathman (1984) \\
Penetracion de la piel, umbral & Esfero de vídrio & 0,4 & 9,7 & Warlow (2012) \\
Penetracion de la piel, umbral & Esfero de acero & 0,4 & 13,1 & Warlow (2012) \\
Penetracion de la piel, umbral & Esfero de latón & 0,4 & 14,5 & Warlow (2012) \\
Penetracion de la piel, umbral & Esfero de plomo & 0,45 & 20,7 & Warlow (2012) \\
Penetracion de la piel, umbral & Balín de plomo, puntiagudo & 0,45 & 20,9 & Warlow (2012) \\
Penetracion de la piel, umbral & Balín de plomo, punto plano & 0,45 & 28,3 & Warlow (2012) \\
Penetracion de la piel, umbral & Balín de plomo, punto hueco & 0,45 & 24,5 & Warlow (2012) \\
& & & &
\end{tabular}


Tabla 2: Valores empíricos de E/a asociados con riesgos de TOS. Algunos estudios usaron ojos de cerdo* y liebret, los cuales tienen una resistencia a la penetración mayor que los ojos humanos (Kennedy et al., 2006):

\begin{tabular}{|c|c|c|c|c|}
\hline$\underline{\text { Riesgo }}$ & Proyectil & Diámetro $(\mathrm{cm})$ & $\mathrm{E} / \mathrm{a}\left(\mathrm{J} \mathrm{cm}^{-2}\right)$ & Fuente \\
\hline $\begin{array}{l}\text { Herida penetrante ocular (HPO), } \\
\text { umbral }\end{array}$ & Esfero de acero & 0,45 & $4,5^{*}$ & Tillet et al. (1962) \\
\hline HPO, umbral & Esfero de plomo & 0,45 & $5,0^{+}$ & Williams y Stewart (1964) \\
\hline HPO, umbral & Esfero de acero & 0,45 & $4,6 \dagger$ & Williams y Stewart (1964) \\
\hline HPO, umbral & Esfero de acero & 0,45 & $4,8 \dagger$ & Powley et al. (2004) \\
\hline HPO, umbral & Esfero de metal & 0,6 & $5,4^{*}$ & Marshall et al. (2011) \\
\hline HPO, umbral & Esfero plástico & 0,6 & $2,9 *$ & Marshall et al. (2011) \\
\hline HPO, umbral & Esfero plástico & 0,6 & $4,3^{*}$ & Marshall et al. (2011) \\
\hline HPO, umbral & Esfero plástico & 0,8 & $4,3 *$ & Marshall et al. (2011) \\
\hline HPO, $50 \%$ probabilidad & varios & varios & 3,20 & Kennedy et al. (2006) \\
\hline HPO, 50\% probabilidad & varios & varios & 2,38 & Duma et al. (2005) \\
\hline Daño retinal, $50 \%$ probabilidad & varios & varios & 3,05 & Duma et al. (2005) \\
\hline $\begin{array}{l}\text { Luxación de la lente intraocular, } \\
50 \% \text { probabilidad }\end{array}$ & varios & varios & 1,92 & Duma et al. (2005) \\
\hline Hifema, $50 \%$ probabilidad & varios & varios & 2,02 & Duma et al. (2005) \\
\hline $\begin{array}{l}\text { Abrasión corneal, } 50 \% \\
\text { probabilidad }\end{array}$ & varios & varios & 0,15 & Duma et al. (2005) \\
\hline
\end{tabular}

Para evaluar E/a de los perdigones de TEC Harseim, se ocuparon valores de masa de $0,7219 \mathrm{~g}$, diámetro de $0,8 \mathrm{~cm}$ y velocidad de salida de $270 \mathrm{~m} \mathrm{~s}^{-1}$, conforme a valores reportados por Labocar (Labocar, 2019). La masa usada es similar a otros valores reportados por informes independientes (Jorquera \& Palma, 2019; Lazo, 2019).

La velocidad de salida representa un valor máximo de proyectiles en vuelo balístico. Cuando el proyectil sale del cañón de un arma, empieza a ser afectado por la resistencia del aire (arrastre), y por lo tanto la velocidad disminuye progresivamente con la distancia. Se utiliza una serie de fórmulas que describen la velocidad de los balines (Allen, 2018) para modelar la velocidad de los perdigones de TEC Harseim a una distancia :

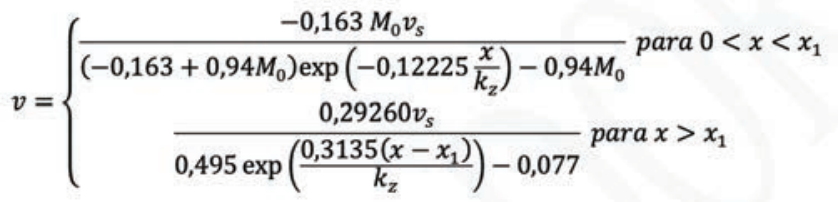

donde

$$
\begin{gathered}
x_{1}=-8,17996 k_{z} \ln \left(\frac{0,70714 M_{0}}{-0,163+0,94 M_{0}}\right) \\
k_{z}=d\left(\frac{\rho_{p}}{\rho_{a t m}}\right)
\end{gathered}
$$

y es la velocidad de salida como número Mach, es la velocidad del sonido $\left(348,95 \mathrm{~m} \mathrm{~s}^{-1}\right.$ a $\left.30^{\circ} \mathrm{C}\right)$ y es la distancia necesaria para que la velocidad del proyectil sea inferior a Mach 0,7. Para estimar el factor de escala se asume un proyectil esférico con diámetro, densidad del proyectil y densidad atmosférica de $1088 \mathrm{~g} \mathrm{~m}^{-3}$ calculada para una temperatura ambiental de $30^{\circ} \mathrm{C}$, humedad relativa de $20 \%$, un punto de rocío de $4,6^{\circ} \mathrm{C}$ y una presión atmosférica de $947,7 \mathrm{hPa}$, que corresponden a condiciones "promedio" de una tarde en Plaza Italia en Santiago (elevación 590 msnm).

Los perdigones de $\mathrm{CBC}$ presentan una masa de $4 \mathrm{~g}$, diámetro de $1,8 \mathrm{~cm}$ y velocidad de salida de $162 \mathrm{~m} \mathrm{~s}^{-1}$ (CBC, 2012). Dado que su velocidad de salida es subsónica (menor que Mach 0,7), se utiliza una fórmula distinta para calcular la velocidad:

$$
v=\frac{0,418 M_{0} v_{s}}{\left(0,418+0,110 M_{0}\right) \exp \left(\frac{0,3135 x}{k_{z}}\right)-0,110 M_{0}} \text { para } x>0
$$

Las fórmulas de Allen (2018) suponen que los proyectiles son esferos perfectos: es decir, que los perdigones tienen dimensiones uniformes, que no se deforman durante el vuelo y que la masa de cada perdigón está uniformemente distribuida. Allen (2018) supone que los esferos individuales no interactúan entre ellos, y se enfatiza que las fórmulas aplican mejor a escopetas sin 
estranguladores (dispositivos de escopeta que alteran la dispersión de perdigones). No se hacen ajustes para los efectos de caída de proyectiles por gravedad ni efectos del viento. No se considera la deformación elástica de los proyectiles al momento del impacto y su efecto en la energía cinética transferida al blanco. Estos supuestos tienen el efecto de exagerar la velocidad y la energía cinética transferida al blanco. Por lo tanto, la velocidad modelada, energía cinética y E/a deben ser considerados como valores máximos.

\section{Resultados}

A continuación, se presentan las curvas de velocidad, energía cinética y E/a para los perdigones de TEC y CBC, junto con una evaluación de las distancias en las que puede ocurrir penetración de piel y HPO. Para evaluar el riesgo de penetración, se usa un valor E/a aproximado de $20 \mathrm{~J} \mathrm{~cm}^{-2}$ (DiMaio, 1998). Para evaluar la probabilidad de HPO, se supone un valor conservador de 3,2 $\mathrm{J} \mathrm{cm}^{-2}$ para provocar HPO en $50 \%$ de los impactos oculares (Kennedy et al., 2006).
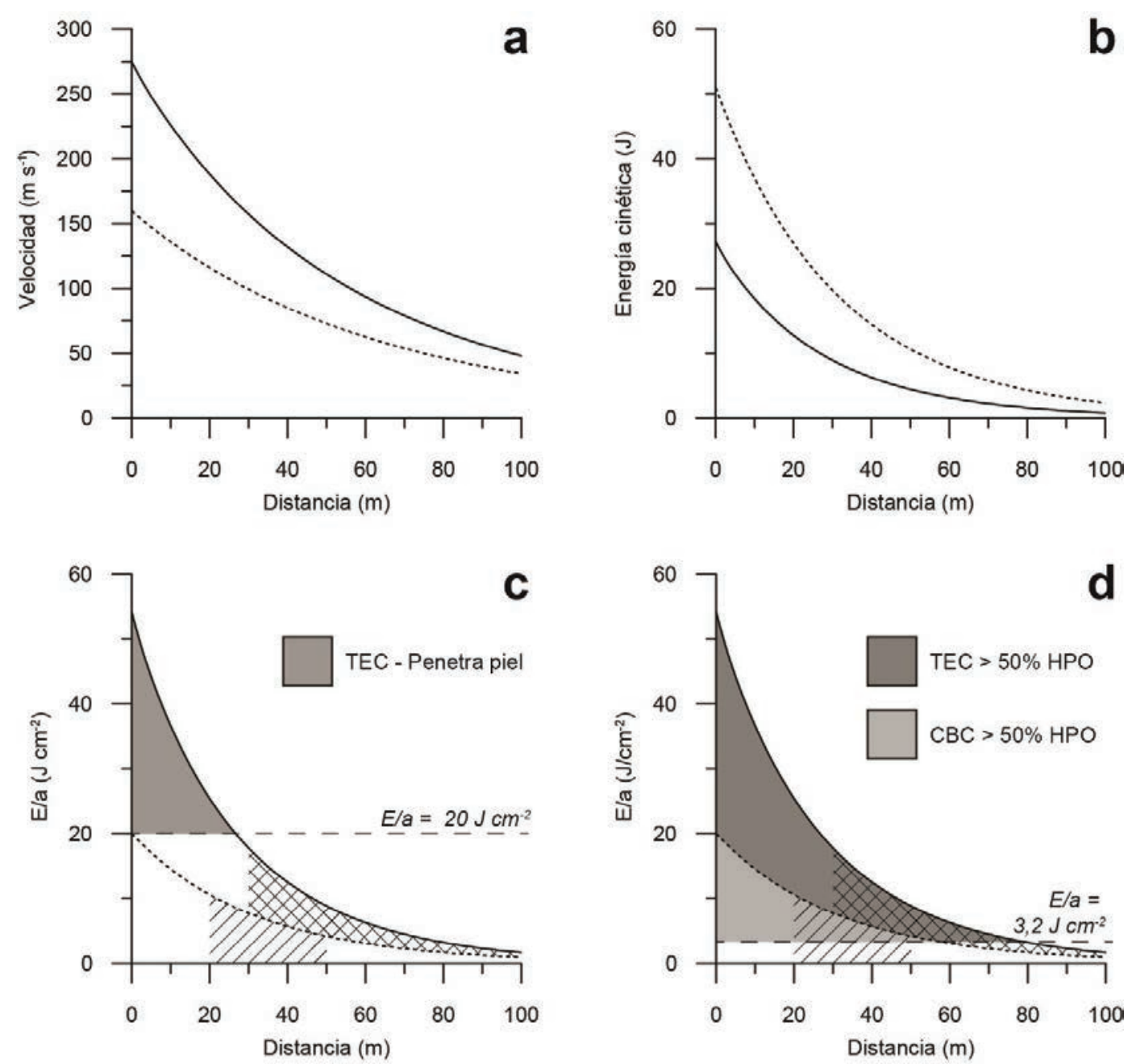

Perdigón TEC

Perdigón CBC

Distancia de uso recomendado TEC

Distancia de uso recomendado $\mathrm{CBC}$

Figura 2: Curvas de velocidad (a), energía cinética (b), y E/a ( c y d) en función de la distancia para los perdigones de TEC y CBC. Los campos con relleno sólido indican el rango de velocidades para cada proyectil que podrían provocar lesiones por penetración de la piel (c) y por heridas penetrantes oculares (HPO; d). Los campos sombreados muestran las distancias de uso recomendadas para los perdigones TEC (Carabineros de Chile, 2012) y CBC (CBC, 2012; Bellolio et al., 2019) 
Pese a la mayor velocidad de salida de los perdigones TEC en comparación a los perdigones $\mathrm{CBC}$, la velocidad balística de los TEC decae más rápidamente, debido a la mayor masa de los proyectiles CBC (Figura 2a). Por la misma razón los perdigones CBC tienen y mantienen una mayor energía cinética que sus contrapartes de TEC (Figura 2b).

Para cualquier distancia dada, los perdigones TEC tienen un E/a mayor que los perdigones CBC. Los perdigones TEC pueden penetrar la piel en una distancia de hasta aproximadamente 28 $\mathrm{m}$, lo cual es menor a lo recomendado por las fuerzas policiales (Carabineros de Chile, 2012). Por otro lado, los perdigones CBC no deberían penetrar la piel (Figura 2c).

Finalmente, existe más de un50\% riesgo de HPO dentro de las distancias de uso recomendadas para ambos proyectiles (Figura $2 \mathrm{~d}$ ). Este riesgo se extiende hasta aproximadamente $80 \mathrm{~m}$ para los perdigones TEC y hasta $60 \mathrm{~m}$ para los perdigones $C B C$.

\section{Discusión y conclusiones}

El presente análisis es el primero que intenta calificar, de manera cuantitativa, las características balísticas de los perdigones menos letales utilizados por las fuerzas de orden pública en Chile. Los resultados indican que-dentro de las distancias sugeridas de uso de las municiones - existe una alta probabilidad de que un impacto en los globos oculares pueda provocar TOS (Figura $2 d$ ). Es decir, bajo el uso adecuado y recomendado de ambas municiones menos letales siempre existe el riesgo de provocar lesiones oculares.
En el caso de los perdigones TEC, el riesgo de penetración de la piel existe solamente para distancias menores que $\sim 28 \mathrm{~m}$. Por lo tanto, los casos de perdigones que han penetrado la piel pueden ser considerados como pruebas de disparos a distancias menores que la distancia mínima recomendada por Carabineros (30 m). El peritaje interno de Carabineros identificó riesgo de penetración de la piel solamente para distancias menores que $10 \mathrm{~m}$ (Carabineros de Chile, 2012). La razón de esta discrepancia podría ser debido a los supuestos considerados en el presente informe, que resultan en una sobreestimación de la distancia de riesgo de penetración. Por otro lado, el carácter cualitativo del informe de Carabineros no descarta la posibilidad de que el peritaje haya subestimado el riesgo representado por los perdigones TEC.

Este análisis se basa en estudios previos de proyectiles similares, pero no idénticos, a los perdigones antidisturbios. Por este motivo, cualquier conclusión con respecto a la balística de estos proyectiles mantendrá un aspecto inductivo hasta que se publiquen pruebas físicas de las medidas, composición, y comportamiento balístico de los perdigones menos letales de las fuerzas policiales chilenas.

La paradoja de los perdigones menos letales es que el riesgo de penetración no permite su uso a distancias en las cuales es posible controlar el agrupamiento de la nube de perdigones. A diferencia de otras armas como rifles y pistolas, las escopetas utilizadas poseen la capacidad de disparar múltiples proyectiles a la vez, los cuales se dispersan entre sí progresivamente con respecto a la distancia (Figura 3). En otras palabras, el tirador de una escopeta no tiene control absoluto de la dispersión de los perdigones, un problema que empeora a mayor distancia.

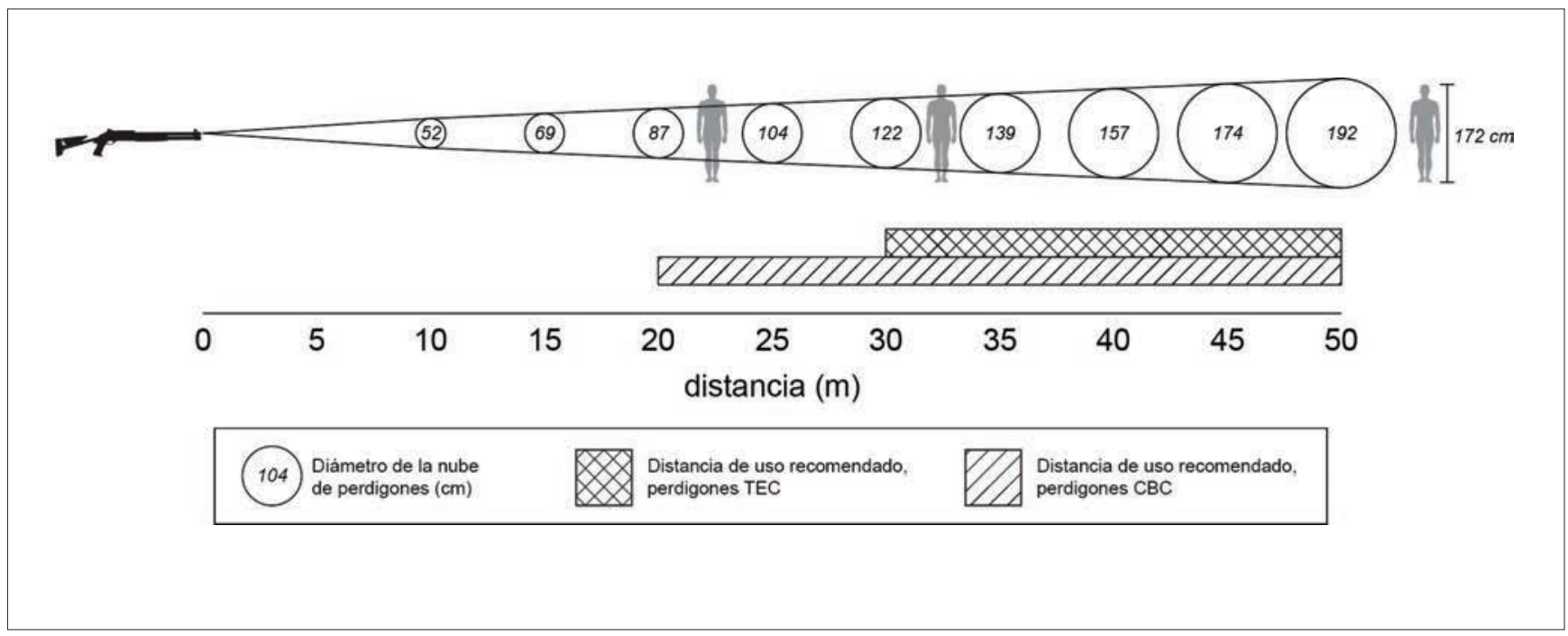

Figura 3: Dispersión de perdigones de plomo con cualquiera escopeta sin estrangulador en función de la distancia (convertido de unidades imperiales de O'Connor, 1961). Adicionalmente, se indican las distancias de uso recomendado para los dos tipos de perdigones estudiados. 
El modelo de dispersión en Figura 3 corresponde a valores estándares derivados empíricamente para perdigones de plomo con escopetas sin estrangulador (O'Connor, 1961). Todavía no es claro si este modelo de dispersión demostrada podría aplicar a perdigones con densidades considerablemente menores, como los de TEC. La dispersión de estas municiones debe ser evaluadas por pruebas físicas para entender cómo su imprecisión y su balística podrían haber afectado la cifra de heridos asociada con su uso.

Se ilustran los efectos cruzados de la poca precisión y balística letal con respecto a perdigones "menos letales" en la respuesta policial a disturbios en el antiguo estado de Jammu y Kashmir en India. Se utilizaron cartuchos de caza menor con 300-600 perdigones de plomo para dispersar a los manifestantes, cuya distancia mínima de uso según el protocolo es de 50 m (David, 2016). Para distancias menores, las municiones son letales, y su uso ha resultado en al menos 17 muertes entre 2016-2017 (Office of the United Nations High Commissioner for Human Rights, 2018). A distancias mayores, la dispersión incontrolable ha sido un factor clave en la alta cifra de TOS registrados en este periodo $(n=728)$. Aumentos abruptos de lesiones oculares por instrumentos de control de multitud ocurrieron también durante la crisis política en Egipto de 2011 (Ahmed \& Zaki, 2013) y en las protestas de los gilets-jaunes en Francia (Chauvin et al., 2019).

Por lo tanto, la alta cifra de TOS en Chile es característica de una tendencia mundial de aumento de casos de lesiones oculares en el contexto de la represión de manifestaciones. Los cartuchos multiproyectiles están involucrados en los casos con mayor incidencia deTOS (en Chile e India). Es notable que estas lesiones no necesariamente requieren el mal uso de las armas según protocolos locales, y por eso los casos de TOS deben contemplarse como un efecto inevitable de la utilización de perdigones antidisturbios. A consecuencia del riesgo inherente de estos perdigones reiterado por el presente estudio, se sugiere reconsiderar el uso continuo de estas municiones en el contexto de control de manifestaciones en Chile.

\section{Contribuciones y reconocimientos}

Se agradece la ayuda de A. Vásquez. y N. Mujica en la revisión del español, y en algunas ediciones para mayor claridad. N. Campillay, D. Kausel, y E. Tobin prestaron ayuda investigativa y en la búsqueda de fuentes primarias. El autor no declara conflictos de interés.

\section{Referencias}

Ahmed SA, \& Zaki RGE. (2013). Forensic Analysis of Ocular Injuries during the 2011 Revolution in Egypt. Forensic Science International 233, 348-354.
Allen, EJ. (2018). Approximate Ballistics Formulas for Spherical Pellets in Free Flight. Defence Technology 14, 1-11.

Bellolio J, Fredes MS, Urbina F, \& Sanhueza E. (2019). Sesión $17^{\text {a }}$ Correspondiente a La 367ª Legislatura, Celebrada En Lunes 25 de Noviembre de 2019, de 16.19 a 18.56 Horas. En Comisión encargada de analizar la procedencia de la acusación constitucional deducida en contra del exministro del Interior y Seguridad Pública, Don Andrés Chadwick Piñera. Cámara de Diputados de Chile. Accedido en https:// www.camara.cl/pdf.aspx?prmID=62570\&prmTIPO=ACTACOMISION el 11 de febrero de 2020.

Carabineros de Chile. (2012). Disparos Con Escopeta Antidisturbios, Con Empleo de Cartuchería Con Perdigón de Goma y Sus Efectos En La Superficie Del Cuerpo Humano, ed. Dirección de Investigación Delictual y Drogas, Departamento de Criminalística. Carabineros de Chile, pp. 1-23. Accedido en https://ciperchile.cl/ wp-content/uploads/INFORME-CARABINEROS_compressed.pdf. el 11 de febrero de 2020.

Chauvin A, Bourges J-L, Korobelnik J-F, Paques M, Lebranchu P, Villeroy F, Chiquet C, Arndt C, Fournier C, \& Villain M. (2019). Ocular Injuries Caused by Less-Lethal Weapons in France. The Lancet 394, 1616-1617.

Companhia Brasileira de Cartuchos. (2012). Informativo Técnico $N^{\circ}$ 38: Cartuchos Para Armas Longas Não Raiadas. Companhia Brasileira de Cartuchos, pp. 1-8. Accedido https://www.cbc.com. br/wp-content/uploads/2018/08/IT-38-Cartuchos-para-ArmasLongas-não-raiadas.pdf. el 18 de enero de 2020

Corgne A. (2019). Identificación de Componentes de Perdigones Por Microscopía Electrónica de Barrido-EDX, ed. Instituto de Ciencias de la Tierra. Universidad Austral de Chile, pp. 1-3. Accedido en https://www.biobiochile.cl/noticias/nacional/region-de-losrios/2019/11/21/estudio-de-la-u-austral-de-chile-ratifica-presencia-de-plomo-en-perdigones-usados-por-carabineros.shtml el 21 de diciembre de 2019.

Cox P, Riveros R, Leiva C, Carvajal Y, Fuentes P, Recasens J, Covarrubias A, Torres F, Ramírez D, Verdugo M, \& Muñoz P. (2019). Análisis Fisicoquímico de Proyectiles Utilizados Durante Las Manifestaciones En Valparaíso y Su Correlación Imagenológica Con Los Cuerpos Extraños Pesquisados Entre El 19 de Octubre y El 15 de Noviembre En El Hospital Carlos Van Buren, ed. Centro de Neurología Traslacional. Universidad de Valparaíso, pp. 1-15. Accedido en http://bit. ly/35qkAjc. el 18 de enero de 2020.

David S. (2016). Use of Pellet Guns for Crowd Control in Kashmir: How Lethal Is 'Non-Lethal'? Indian Journal of Medical Ethics 2, 1-3. 
De Brito D, Challoner KR, Sehgal A, \& Mallon W. (2001). The Injury Pattern of a New Law Enforcement Weapon: The Police Bean Bag. Annals of Emergency Medicine 38, 383-390.

DiMaio VJM. (1981). Penetration and Perforation of Skin by Bullets and Missiles. A Review of the Literature. The American Journal of Forensic Medicine and Pathology 2, 107-110.

DiMaio VJM. (1998). Gunshot Wounds: Practical Aspects of Firearms, Ballistics, and Forensic Techniques. 2nd ed. CRC Press, Boca Raton.

Duma SM, Ng TP, Kennedy EA, Stitzel JD, Herring IP, \& Kuhn F. (2005). Determination of Significant Parameters for Eye Injury Risk from Projectiles. The Journal of Trauma 59, 960-964.

Figueroa N, y Romero N. (2020). Carabineros No Entrega Información Sobre Composición y Protocolo Para El Uso de Nuevos Perdigones. El Desconcierto, 22 de enero de 2020. Accedido en https://www. eldesconcierto.cl/2020/01/22/carabineros-no-entrega-informacion-sobre-composicion-y-protocolo-para-el-uso-de-nuevos-perdigones/. el 23 de enero de 2020

Haar RJ, lacopino V, Ranadive N, Dandu M, \&Weiser SD. (2017). Death, Injury and Disability from Kinetic Impact Projectiles in Crowd-Control Settings: A Systematic Review. BMJ Open 7, pp. e018154.

Jorquera PE, \& Palma RH. (2019). Estudio de Perdigón: Infore Final (UTO), ed. Departamento de Ingeniería Mecánica, Facultad de Ciencias Físicas y Matemáticas. Universidad de Chile, pp. 1-17. Accedido en https://radio.uchile.cl/wp-content/uploads/2019/11/ INFORME-UTOSInfFinv1.png.pdf. el 16 de noviembre de 2019

Journée F-A. (1907). Rapport Entre La Force Vive Des Balles et La Gravité Des Blessures Qu'elles Peuvent Causer. Rev. d'Artilleries 70, 81-120.

Kennedy EA, Ng TP, \& Duma SM. (2006). Evaluating Eye Injury Risk of Airsoft Pellet Guns by Parametric Risk Functions. Biomedical Sciences Instrumentation 42, 7-12.

Labocar. (2019). Resumen Ejecutivo Nro. 03, ed. Sección Criminalística Iquique. Carabineros de Chile, pp. 1-9. Accedido https:// ciperchile.cl/wp-content/uploads/Informe-Labocar.pdf. el 23 de noviembre de 2019.

Lazo C. (2019). Análisis de Perdigones Utilizados Por Carabineros En La Ciudad de Valdivia, ed. Instituto de Ciencias de la Tierra. Universidad Austral de Chile, pp. 1-3. Accedido el https://issuu. com/rioenlinea/docs/comunicado_1_. el 8 de febrero de 2020
Mahajna A, Aboud N, Harbaji I, Agbaria A, Lankovsky Z, Michaelson M, Fisher D, \& Krausz MM. (2002). Blunt and Penetrating Injuries Caused by Rubber Bullets during the Israeli-Arab Conflict in October, 2000: A Retrospective Study. The Lancet 359, 1795-1800.

Marshall JW, Dahlstrom DB, \& Powley KD. (2011). Minimum Velocity Necessary for Nonconventional Projectiles to Penetrate the Eye: An Experimental Study Using Pig Eyes. American Journal of Forensic Medicine and Pathology 32, 100-103.

Mattoo BN, Wani AK, \& Asgekar MD. (1974). Casualty Criteria for Wounds from Firearms with Special Reference to Shot Penetration-Part II. Journal of Forensic Science 19, 585-589.

Millar R, Rutherford WH, Johnston S, \& Malhotra VJ. (1975). Injuries Caused by Rubber Bullets: A Report on 90 Patients. British Journal of Surgery $62,480-486$.

Ministerio del Interior y Seguridad Pública. (2019). Uso de La Fuerza: Actualiza Instrucciones Al Respecto: Circular Número 1832. Diario Oficial de la República de Chile, Santiago. Accedido en https://www.diariooficial.interior.gob.cl/publicaciones/2019/03/04/42295/01/1556120. pdf. el 16 de noviembre de 2019

O'Connor J. (1961). Complete Book of Rifles and Shotguns. Outdoor Life, New York.

Office of the United Nations High Commissioner for Human Rights. (2018). Report on the Situation of Human Rights in Kashmir: Developments in the Indian State of Jammu and Kashmir from June 2016 to April 2018, and General Human Rights Concerns in Azad Jammu and Kashmir and Gilgit-Baltistan, ed. Office of the United Nations High Commissioner for Human Rights. United Nations, pp. 1-49. Accedido en https://www.ohchr.org/Documents/ Countries/IN/DevelopmentsInKashmirJune2016ToApril2018.pdf. 11 de febrero de 2020 .

Powley KD, Dahlstrom DB, Atkins VJ, \& Fackler ML. (2004). Velocity Necessary for a BB to Penetrate the Eye: An Experimental Study Using Pig Eyes. The American Journal of Forensic Medicine and Pathology 25, 273-275.

Rathman GA. (1987). The effect of shape on BB and pellet penetration. Journal of the Forensic Science Society 24, 393.

Rocke L. (1983). Injuries caused by plastic bullets compared with those caused by rubber bullets. The Lancet 321, 919-920. 


\section{Reynhout}

Rodríguez A, Cortés D, Cavieres I, Campos MA, Vergara MJ, Peredo D, López M, \& Morales S. (2019). Informe Trauma Ocular, ed. Unidad de Trauma Ocular. Hospital del Salvador, pp. 1-13. Accedido en https://www.sochiof.cl/noticias-detalle/92/ informe-unidad-trauma-ocular-hospital-del-salvador el 18 de enero de 2020.

Tillett CW, Rose HW, \& Carl Herget. (1962). High-Speed Photographic Study of Perforating Ocular Injury by the BB. American Journal of Ophthalmology 54, 675-688.
United Nations Regional Centre for Peace, Disarmament and Development in Latin America and the Caribbean. (2016). Armas Menos Letales En América Latina y El Caribe: Retos y Oportunidades, ed. Centro Regional de las Naciones Unidas para la Paz, el Desarme y el Desarrollo en América Latina y el Caribe. Naciones Unidas, pp. 1-38. Accedido http:// www.unlirec.org/Documents/AML_ALC.pdf el 11 de febrero de 2020.

Warlow T. (2012). Firearms, the Law, and Forensic Ballistics. 3rd ed. CRC Press, Boca Raton.

Williams RL, and Stewart GM. (1964). Ballistic Studies in Eye Protection. American Journal of Ophthalmology 58, 453-464. 